\title{
Asymptotic dimension and uniform embeddings
}

\author{
Światosław R. Gal*
}

\begin{abstract}
We study uniform embeddings of metric spaces, which satisfy some asymptotic tameness conditions such as finite asymptotic dimension, finite Assouad-Nagata dimension, polynomial dimension growth or polynomial growth, into function spaces.

We show how the type function of a space with finite asymptotic dimension estimates its Hilbert (or any $\ell^{p}$-) compression. In particular, we show that the spaces of finite asymptotic dimension with linear type (spaces with finite Assouad-Nagata dimension) have compression rate equal to one.

We show, without the extra assumption that the space has the doubling property (finite Assouad dimension), that a space with polynomial growth has polynomial dimension growth and compression rate equal to one.

The method used allows us to obtain a lower bound on the compression of the lamplighter group $\mathbb{Z} \imath \mathbb{Z}$, which has infinite asymptotic dimension.
\end{abstract}

Mathematics Subject Classification (2000). Primary 20F69, 20F65, 20H15; Secondary 20E22, 54F45, 51F99.

Keywords. Uniform embeddings, metric spaces, asymptotic dimension, Assouad-Nagata dimension, Hilbert space compression, coarse geometry.

\section{Introduction}

The study of embeddings of metric spaces into function spaces (where Hilbert space plays a prominent role) was introduced by M. Gromov in [Gro93, Section 7.E]. It appeared to be a fundamental tool in geometric group theory since G. Yu proved that a group admitting a uniform embedding with respect to the word metric into a Hilbert space satisfies the Novikov Conjecture on homotopy equivariance of higher signatures [Yu00] (which was already predicted by M. Gromov).

In [Gro93, p. 29] Gromov also introduced a large scale twin of the topological covering dimension, the asymptotic dimension. As noticed by N. Higson and

\footnotetext{
*Partially supported by Swiss FNRS Grant 20-109130 U 00998 and 200020-109175 and Polish Grant N201 012 32/0718.
} 
J. Roe [HR00] spaces of finite asymptotic dimension have property A of G. Yu, and thus, in particular, they uniformly embed into $\ell^{p}$ spaces. This idea already appears in [Yu98, Section 6].

In [GK04] E. Guentner and J. Kaminker initiated the quantitative study of such embeddings (they defined the compression rate of such an embedding). This topic was developed recently in a number of papers [AGS04], [CTV05], [SV06], [Tes06], [Tes07], [ADS06].

In this paper we compare three (families of) profiles associated to metric spaces.

- The type function $D_{k}$, which is essentially the one introduced by M. Gromov in [Gro93] (the two definitions will be compared in Section 3.1). The function $D_{k}$ is well defined (finite) under the assumption that the space in question has asymptotic dimension at most $k$.

- The family of functions $\delta_{p}$ which are defined for arbitrary metric space, and still encode interesting information when the space has infinite asymptotic dimension in a tame way (such as polynomial dimension growth defined by A. Dranishnikov [Dra06]). In Section 4.3 we will estimate those functions for the wreath product $\mathbb{Z} \imath \mathbb{Z}$ (the lamplighter group).

- The profiles $\epsilon_{p}$ being a quantitive measure of how well the space satisfies property $A$ of $\mathrm{G}$. Yu.

These profiles will be used to estimate (from below) the compression rate of spaces satisfying some tameness properties such as finite asymptotic dimension, finite AssouadNagata dimension, polynomial dimension growth or polynomial growth.

Section 3 is devoted to the proof of the main result, which is the comparison between these three families of functions:

$$
\frac{4}{\epsilon_{p}} \geq \delta_{p} \geq\left(\frac{1}{\sqrt{k}}\right)^{p} D_{k}^{-1} .
$$

As a straightforward corollary we obtain a lower bound for the Hilbert compression of spaces of finite asymptotic dimension in terms of its type function (Theorem 1.1.1). In particular, we will show that the spaces of finite asymptotic dimension with linear type (spaces with finite Assouad-Nagata dimension) have compression rate equal to one.

In Section 4 we will apply this result to some spaces with infinite asymptotic dimension. We will show that a space with polynomial growth has polynomial dimension growth and compression rate equal to one (Theorem 4.1.4). A weaker result, with the extra assumption that the space has the doubling property (finite Assouad dimension), follows from the celebrated Assouad Embedding Theorem [Ass83].

Finally, in Section 4.3, more subtle estimates of $\delta_{p}$ will give a lower bound for the Hilbert compression of $\mathbb{Z} \imath \mathbb{Z}$. 
1.1. The type function and compression. The type function $D_{k}$ (cf. [Gro93, p. 29]) of a metric space $X$ is defined as follows (we will recall this definition in detail in Section 3.1 as Definition 3.1.3). $D_{k}(L)$ is the infimum of those $S$ such that there exists an open cover $\mathfrak{U}$ of $X$ by sets of diameter at most $S$, the multiplicity of $\mathcal{U}$ is at most $k+1$ and, for every $x \in X$, the ball $B_{L}(x)$ of radius $L$ around $x$ is contained in some set from $\mathcal{U}$. The space $X$ is said to have asymptotic dimension at most $k$ if $D_{k}(L)<\infty$ for all positive $L$.

Theorem 1.1.1. Let $(X, d)$ be a space of bounded geometry, asymptotic dimension at most $k$ and with type function $D_{k}$. Let $u$ be any non-decreasing function such that the Riemann-Stieltjes integral

$$
\int_{c}^{\infty} \frac{d u(t)^{p}}{t^{p}}<\infty
$$

converges. Then there exists a Lipschitz map $\theta: X \rightarrow \ell^{p}$ such that

$$
d(x, y) \geq D_{k}(t) \Rightarrow\|\theta(x)-\theta(y)\|_{p} \geq u(t) .
$$

In particular, one can take $u(t)=t \cdot(\log t)^{-(1+a) / p}$ for any $a>0$ (see Example 2.4.4). Another form of the condition (1) appears in [Tes07] as condition $\left(C_{p}\right)$ (we will show the equivalence in Section 2.5).

The proof of Theorem 1.1.1 will be given on p. 78 after Corollary 3.2.2.

If $(X, d)$ is a group with the word metric, then the type function grows at least linearly since the diameter of a ball of radius $L$ is at least $2\lfloor L\rfloor$. The same is true for quasi-geodesic spaces.

The spaces with linear type function are also known as spaces with finite AssouadNagata dimension [Ass82], [LS05] or spaces with Higson property.

Example 1.1.2. The class of spaces with linear type function contains trees and Lobačevskiǐ hyperbolic spaces [Gro93, p. 29].

Unfortunately, the most common way to show that a space has linear type function is to embed it into a product of trees and/or hyperbolic spaces. M. Bonk and O. Schramm [BS00] showed that every Gromov hyperbolic group admits a quasiisometric embedding into a Lobačevskiǔ hyperbolic space. Alternatively, S. Buyalo and V. Schroeder [BS05] showed that such a group embeds quasi-isometrically in a product of a finite number of trees.

Also Coxeter groups have linear type function as they can be embedded quasiisometrically in a product of (locally finite) regular trees [Jan02].

Another example is given by amenable Baumslag-Solitar groups defined by the presentation $B S_{m}^{1}=\left\langle a, t \mid t a t^{-1}=a^{m}\right\rangle$ (where $m>1$ is any integer). They embed 
quasi-isometrically into the product of an $(m+1)$-regular tree and a Lobačevskiǔ space [FM98].

Recently, P. Nowak [Now07] found the first examples of groups with finite asymptotic dimension (even of asymptotic dimension one) with nonlinear type function. Later G. Arzhantseva, C. Druţu and M. Sapir [ADS06] constructed groups of asymptotic dimension 2 having very small compression, therefore with very large type functions.

Acknowledgements. The author warmly thanks Piotr Nowak, Nicolas Monod and Romain Tessera for inspiring discussions. The author is also grateful to Pierre de la Harpe and the University of Geneva for hospitality during the final work on the manuscript.

1.2. Notation and preliminaries. Let $f, g: \mathbb{R}_{+} \rightarrow \mathbb{R}_{+}$be two weakly monotone functions. We write $f \succeq g$ if there exist positive constants $C$ and $D$ such that $f(t) \geq C g(D t)$ for sufficiently large $t>0$. We write $f \sim g$ if $f \succeq g$ and $f \preceq g$.

If $f$ is a non-decreasing (resp. non-increasing) positive function, then by $f^{-1}(t)$ we mean inf $f^{-1}([t, \infty))$ (resp. sup $\left.f^{-1}((0, t])\right)$.

Definition 1.2.1. A metric space is said to have bounded geometry if for every $R \geq 0$ there exists $C<\infty$ such that every ball of radius $R$ contains at most $C$ points.

Most of this work remains true for a wider class of spaces namely measure-metric spaces with bounded geometry (cf. [Tes07]).

By $\mathbb{S}_{1}\left(\ell^{p} X\right)$ we will denote the unit sphere in $\ell^{p} X$. In this paper we will consider $\ell^{p}$ spaces only with $1 \leq p<\infty$.

\section{Uniform embeddings in $\ell^{p}$ spaces}

2.1. Property $A$ and the associated profile. In this section we introduce a quantitative description of property A of G. Yu which will be subsequently used in the proof of Theorem 1.1.1. Other definitions were recently proposed by P. Nowak [Now07, Definition 3.2] and R. Tessera [Tes07, Definition 4.1].

Definition 2.1.1. Let $\xi: X \times X \rightarrow \mathbb{R}$ be a kernel. We will write $(x, y) \mapsto \xi_{x}(y)$. Define

(1) $S(\xi):=\sup \left\{d(x, y) \mid \xi_{x}(y) \neq 0\right\}$,

(2) $\varepsilon(\xi ; p):=\sup \left\{\frac{\left\|\xi_{x}-\xi_{y}\right\|_{p}}{d(x, y)} \mid x \neq y\right\}$.

Remark 2.1.2. A map $\xi: X \rightarrow \ell^{p}(X)$ is $\varepsilon(\xi ; p)$-Lipschitz, and $\varepsilon(\xi ; p)$ is the best Lipschitz constant. 
Definition 2.1.3. For a space $X$ define

$$
\epsilon_{X ; p}(S):=\inf \left\{\varepsilon(\xi ; p) \mid \xi: X \rightarrow \mathbb{S}_{1}\left(\ell^{p} X\right), S(\xi) \leq S\right\} .
$$

In particular, for any $S>0$, it is possible to find a map $\xi: X \rightarrow \mathbb{S}_{1}\left(\ell^{p} X\right)$ with $S(\xi) \leq S$ and $\varepsilon(\xi ; p)<2 \epsilon_{X ; p}(S)$. Notice that $\epsilon_{X ; p}$ is a non-increasing function. We will write $\epsilon_{X ; p}=: \epsilon_{p}$ if it does not lead to ambiguity. We will call $\epsilon_{p}$ the $p$ th-profile associated with property $A$.

We do not recall the original definition of the property A due to G. Yu [Yu00, Definition 2.1]. Instead we give an equivalent formulation due to N. Higson and J. Roe [HR00, Lemma 3.5].

Definition 2.1.4. A discrete metric space $X$ of bounded geometry has property A if for any $R>0$ and $\epsilon>0$ there exists $\xi: X \rightarrow \mathbb{S}_{1}\left(\ell^{1} X\right)$ with $S(\xi)<\infty$ and such that $\left\|\xi_{x}-\xi_{y}\right\| \leq \epsilon$ provided $d(x, y) \leq R$.

Proposition 2.1.5. If inf $\epsilon_{X ; 1}=0$ then $X$ has property A.

Proof. $\xi: X \rightarrow \mathbb{S}_{1}\left(\ell^{p} X\right)$ with $S(\xi)<\infty$ and $\varepsilon(\xi ; 1)=\epsilon / R$ satisfies the condition in the above definition.

To prove the opposite implication we need a mild assumption that the space is uniformly discrete (which can be always realized in a quasi-isometry class of the metric).

Definition 2.1.6. A metric space $(X, d)$ is uniformly discrete if zero is an isolated value of the metric.

An example of uniformly discrete space is a vertex set of a graph (with unit length edges) with an induced metric, e.g. a discrete group with a word metric.

Proposition 2.1.7. Assume that a uniformly discrete space $X$ has property A. Then inf $\epsilon_{X ; 1}=0$.

Proof. By hypothesis we can find $r>0$ such that $d(x, y)<r$ implies $x=y$. Assume that $\left\|\xi_{x}-\xi_{y}\right\| \leq \epsilon \cdot r$ for $d(x, y) \leq 2 / \epsilon$. Then $\xi: X \rightarrow \ell^{1}(X)$ is $\epsilon$-Lipschitz. zero).

We are interested in the asymptotic behavior of $\epsilon_{X ; p}$ (how fast it does converge to

Remark 2.1.8. Let $d(x, y)=2 S(\xi)$. Then $\left\|\xi_{x}-\xi_{y}\right\|=2$. Therefore $\varepsilon(\xi ; p) \geq$ $S(\xi)^{-1}$ for all such $\xi$ and $\epsilon_{p}(S) \geq S^{-1}$. This demonstrates that we cannot expect faster decay of $\epsilon_{p}(S)$ than $S^{-1}$ for a general metric space. 
More precisely, let us make a reasonable assumption on the metric space. We assume that there exists $M$ such that for $\delta$ sufficiently large there are $x, y \in X$ such that $\delta<d(x, y)<M \delta$.

Under the above assumption $\epsilon_{p}(S) \leq(M S)^{-1}$. Indeed, if $d(x, y)>2 S$ and $S(\xi) \leq S$, then $\xi_{x}$ and $\xi_{y}$ have disjoint supports and $\left\|\xi_{x}-\xi_{y}\right\|=2$. Thus if $2 M S>d(x, y)>2 S$, then $\left\|\xi_{x}-\xi_{y}\right\| / d(x, y)>(M S)^{-1}$.

Example 2.1.9. Let $V$ be a vertex set (with the induced metric) of any simplicial tree. Then $\epsilon_{V ; p}(S) \geq(2 S)^{-1 / p}$. Indeed, fix a point $\omega$ in the boundary of the tree. Let $\xi_{x}(y)=S^{-1 / p}$ when $y$ is at distance at most $S$ from $x$ in the direction of $\omega$. Then $\xi: V \rightarrow \mathbb{S}_{1}\left(\ell^{p} V\right)$ is $(2 S)^{-1 / p}$-Lipschitz [DJ99, Proposition 1].

The above estimate is sharp only for $p=1$. The following example shows the optimal estimate. Although it is a direct corollary of Corollary 3.2.2 in the case where the tree is uniformly locally finite (has bounded geometry), we find it instructive to do the proof by hand in full generality.

Proposition 2.1.10. Let $V$ be a vertex set (with the induced metric) of any simplicial tree. For any $S \geq 1$ one can construct $\xi: V \rightarrow \mathbb{S}_{1}\left(\ell^{p} V\right)$ with $S(\xi) \leq S$ and such that $\xi$ is $8 S^{-1}$-Lipschitz.

Proof. Define $\zeta_{x}(z)=\max (S+2-|S-2 d(x, z)|, 0)$ if the geodesic ray from $x$ towards $\omega$ goes through $z$ and $\zeta_{x}(z)=0$ otherwise. Then

$$
\left\|\zeta_{x}\right\|_{p}>\left(2 \int_{0}^{S / 2}(2 t)^{p} d t\right)^{1 / p}=\left(\frac{S^{p+1}}{p+1}\right)^{1 / p}=\frac{S^{1+1 / p}}{(p+1)^{1 / p}},
$$

and if $d(x, y)=1$, then $\left\|\zeta_{x}-\zeta_{y}\right\|_{p}^{p}=2^{p}(2\lfloor S / 2\rfloor+2)$. Thus $\xi_{x}=\zeta_{x} /\left\|\zeta_{x}\right\|_{p}$ satisfies

$$
\left\|\xi_{x}-\xi_{y}\right\|_{p}<2\left(\frac{2\lfloor S / 2\rfloor+2}{S}\right)^{1 / p}(1+p)^{1 / p} S^{-1} \leq 8 S^{-1},
$$

for $d(x, y)=1$.

2.2. Quasi-isometry invariance. Let $f: X \rightarrow Y$ be a map. If $X$ and $Y$ are equipped with metrics $d_{X}$ and $d_{Y}$ we define the compression $\rho_{f}$ of $f$ as the greatest non-decreasing function such that

$$
d_{Y}\left(f(x), f\left(x^{\prime}\right)\right) \leq \rho_{f}\left(d_{X}\left(x, x^{\prime}\right)\right)
$$

for all $x, x^{\prime} \in X$. 
Proposition 2.2.1. Let $f: X \rightarrow Y$ be an L-Lipschitz map. Then $\epsilon_{Y ; p}\left(\rho_{f}(S)\right) \leq$ $L \cdot \epsilon_{X ; p}(3 S)$.

Proof. Choose a map $s: Y \rightarrow X$ such that $f(s(y)) \in B(y, 2 \operatorname{dist}(y, f(X))$. This can be done since obviously $f^{-1}(B(y, 2 \operatorname{dist}(y, f(X)))$ is not empty. Notice that

$$
d(f(x), f(s(y))) \leq d(f(x), y)+d(f(s(y)), y) \leq 3 d(f(x), y),
$$

while $d(f(s(y)), y) \leq 2 \operatorname{dist}(f(X), y) \leq 2 d(f(x), y)$. In particular, $f(s(y))=y$ if $y \in f(X)$.

For $\xi: Y \rightarrow \ell^{p}(Y)$ we define $\sigma: X \rightarrow \ell^{p}(X)$ by the formula

$$
\sigma_{x}(z):=\left(\sum_{s(y)=z}\left|\xi_{f(x)}(y)\right|^{p}\right)^{1 / p} .
$$

It remains to show that

$$
\begin{aligned}
\left\|\sigma_{x}\right\|_{p} & =\left\|\xi_{f(x)}\right\|_{p} \\
\left\|\sigma_{x}-\sigma_{x^{\prime}}\right\|_{p} & \leq\left\|\xi_{f(x)}-\xi_{f\left(x^{\prime}\right)}\right\|_{p}
\end{aligned}
$$

and

$$
\rho_{f}(S(\sigma)) \leq 3 S(\xi)
$$

Indeed,

$$
\begin{gathered}
\left\|\sigma_{x}\right\|_{p}^{p}=\sum_{z \in X} \sum_{s(y)=z}\left|\xi_{f(x)}(y)\right|^{p}=\sum_{y \in Y}\left|\xi_{f(x)}(y)\right|^{p}=\left\|\xi_{f(x)}\right\|_{p}^{p}, \\
\left\|\sigma_{x}-\sigma_{x^{\prime}}\right\|_{p}^{p}=\sum_{z \in X}\left(\left(\sum_{s(y)=z}\left|\xi_{f(x)}(y)\right|^{p}\right)^{1 / p}-\left(\sum_{s(y)=z}\left|\xi_{f\left(x^{\prime}\right)}(y)\right|^{p}\right)^{1 / p}\right)^{p}
\end{gathered}
$$

(by the triangle inequality in $\ell^{p}\left(s^{-1}(z)\right)$ )

$$
\begin{aligned}
& \leq \sum_{z \in X} \sum_{S(y)=z}\left|\xi_{f(x)}(y)-\xi_{f\left(x^{\prime}\right)}(y)\right|^{p}=\left\|\xi_{f(x)}-\xi_{f\left(x^{\prime}\right)}\right\|_{p}^{p} \\
\rho_{f}(S(\sigma)) & =\sup \left\{\rho_{f}\left(d_{X}(x, z)\right) \mid \sigma_{x}(z) \neq 0\right\} \\
& \leq \sup \left\{d_{Y}(f(x), f(z)) \mid \sigma_{x}(z) \neq 0\right\} \\
& \leq \sup \left\{d_{Y}(f(x), f(s(y))) \mid \xi_{f(x)}(y) \neq 0\right\} \\
& \leq \sup \left\{3 d_{Y}(f(x), y) \mid \xi_{f(x)}(y) \neq 0\right\} \\
& \leq \sup \left\{3 d_{Y}(u, y) \mid \xi_{u}(y) \neq 0\right\}=3 S(\xi)
\end{aligned}
$$

A consequence of the previous proposition is the quasi-isometry invariance of $\epsilon_{X ; p}$. 
Corollary 2.2.2. Let $X$ and $Y$ be uniformly discrete metric spaces. Let $f: X \rightarrow Y$ be a quasi-isometry. Then

$$
\epsilon_{X ; p} \sim \epsilon_{Y ; p} .
$$

In particular, if $G$ is a finitely generated group equipped with a word metric, then the asymptotic behaviour of $\epsilon_{G ; p}$ is independent of the chosen generating set.

Proof. Observe that if the target space is uniformly discrete, then any large scale Lipschitz map is in fact Lipschitz.

From Proposition 2.2.1 we get an immediate

Corollary 2.2.3. Let $X \subseteq Y$ be a subspace with the induced metric. Then

$$
\epsilon_{X ; p} \preceq \epsilon_{Y ; p} .
$$

2.3. The dependence on $p$. For the proofs of the estimates of this section the reader may consult [BL00] or easily adjust the proofs from the original paper [Maz29].

Consider the map $M: \mathbb{S}_{1}\left(\ell^{q} X\right) \rightarrow \mathbb{S}_{1}\left(\ell^{p} X\right)$ (called the Mazur map) defined by the formula

$$
(M f)(x)=|f(x)|^{q / p-1} f(x) .
$$

This map is $q / p$-Lipschitz if $p \leq q$. Thus we obtain

Corollary 2.3.1. Assume that $p \leq q$. Then

$$
\epsilon_{p} \leq q / p \cdot \epsilon_{q} .
$$

Lemma 2.3.2. Assume that $\epsilon_{q}(S) \leq \varphi(S) S^{\alpha / q}$, where $\varphi$ does not depend on $q$. Then

$$
\epsilon_{p}(S) \leq \frac{e^{\alpha}}{p} \varphi(S) \log (S),
$$

for $S \geq e^{p}$.

Proof. This follows from Corollary 2.3 .1 by putting $q=\log S$.

On the other hand, if $p \geq q$ the Mazur map is only Hölder with exponent $p / q$.

Thus, even if $\epsilon_{1}(S) \preceq S^{-1}$ (i.e. $\epsilon_{1}$ has the fastest possible decay, cf. Remark 2.1.8), the estimate on $\epsilon_{p}$ coming from the Mazur map $\left(\epsilon_{p}(S) \preceq S^{-1 / p}\right)$ is usually far from sharp (cf. Example 2.1.9 and Proposition 2.1.10). 


\subsection{The $\ell^{p}$-compression}

Definition 2.4.1 ([Gro93]). Let $X, Y$ be metric spaces. A map $f: X \rightarrow Y$ is a coarse embedding (uniform embedding) if there exist non-decreasing functions $\rho_{-}, \rho_{+}:[0, \infty) \rightarrow[0, \infty)$ satisfying

(1) $\rho_{-}\left(d_{X}(x, y)\right) \leq d_{Y}(f(x), f(y)) \leq \rho_{+}\left(d_{X}(x, y)\right)$ for all $x, y \in X$,

(2) $\lim _{t \rightarrow \infty} \rho_{-}(t)=+\infty$.

The smallest function $\rho_{+}$one can choose is called the dilation of $f$.

If the space $X$ is quasi-geodesic (as for example the Cayley graph of a group) then one can take $\rho_{+}$to be an affine function. The main quantitative interest is how big can one choose $\rho_{-}$.

The Hilbert space compression rate of a metric space $X$ was introduced by E. Guentner and J. Kaminker [GK04] and it is the supremum of $\alpha$ such that $X$ admits a coarse embeddings into the Hilbert space with lower bound $\rho_{-}(t) \succeq t^{\alpha}$.

Below we consider embeddings in an $\ell^{p}$ space for any $p \geq 1$.

Proposition 2.4.2. Let $X$ be a metric space. Let $f:[c, \infty) \rightarrow \mathbb{R}$ be a non-decreasing left-continuous function. Assume that a measurable field of maps

$$
[c, \infty) \times X \ni(S, x) \mapsto \xi_{x}^{S} \in \mathbb{S}_{1}\left(\ell^{p} X\right)
$$

satisfies $S\left(\xi^{S}\right) \leq S$ and the Riemann-Stieltjes integral

$$
\int_{c}^{\infty} \varepsilon\left(\xi^{S}, p\right)^{p} d(f(S))^{p}=: L
$$

is convergent $(L<\infty)$. Then the map $\theta(x):=\xi(x)-\xi\left(x_{0}\right)$ (where $x_{0}$ is an arbitrary reference point) is an $L^{1 / p}$-Lipschitz map $X \rightarrow L^{p}\left([c, \infty), d f^{p}\right) \otimes \ell^{p}(X)$ which compression $\rho_{-}$is asymptotically bounded by $f$, more precisely

$$
\rho_{-}(d) \geq 2 f(d / 2)-2 f(c) .
$$

Proof. Step 1 ( $\theta$ is $L^{1 / p}$-Lipschitz). From the definition of the norm in the space $L^{p}\left([c, \infty), d f^{p}\right) \otimes \ell^{p}(X)$ we have

$$
\begin{aligned}
\|\theta(x)-\theta(y)\|_{p}^{p} & =\int_{c}^{\infty}\left\|\xi_{x}^{S}-\xi_{y}^{S}\right\|_{p}^{p} d f(S)^{p} \\
& \leq\left(\int_{c}^{\infty} \varepsilon\left(\xi^{S}, p\right)^{p} d f(S)^{p}\right) d(x, y)^{p}=L d(x, y)^{p} .
\end{aligned}
$$


Step 2 (bound on the compression). Assume that $d(x, y)>d$. Since $\operatorname{supp}\left(\xi_{x}^{S}\right) \subset$ $B_{S}(x)$ we have that $\left\|\xi_{x}^{S}-\xi_{y}^{S}\right\|_{p}=2$ if $2 S \leq d$. What follows,

$$
\begin{aligned}
\|\theta(x)-\theta(y)\|_{p}^{p} & \geq \int_{c}^{d / 2} 2^{p} d f(S)^{p} \\
& =2^{p}\left(f(d / 2)^{p}-f(c)^{p}\right) \geq 2^{p}(f(d / 2)-f(c))^{p} .
\end{aligned}
$$

The claim follows by the continuity of $f$ on the left.

Corollary 2.4.3. Let $X$ be a metric space. Let $f:[c, \infty) \rightarrow R$ be a non-decreasing function such that

$$
\int_{c}^{\infty} \epsilon_{p}(S)^{p} d f(S)^{p}<\infty
$$

Then there exists a uniform embedding $\theta: X \rightarrow \ell^{p}$ such that the compression $\rho_{-}$ satisfies $\rho_{-} \succeq f$.

Proof. The assumption in Proposition 2.4.2 that $f$ is left-continuous was made only to get a precise bound on the compression. Replacing $f$ by $g(t):=\lim _{s \backslash t} f(s)$ makes the function $g$ left-continuous and does not change the value of the integral. On the other hand, $f \sim g$ with constants arbitrary close to one.

Taking sufficiently small subdivision of $[c, \infty]$ one constructs a piecewise constant (thus measurable) field $\xi$ with the property $\varepsilon\left(\xi^{S} ; p\right)<2 \epsilon_{p}(S)$ and $S\left(\xi^{S}\right) \leq S$.

One can always approximate $g$ by a piecewise constant $h$ with the same asymptotic behavior. In this case $L^{p}\left([c, \infty), d h^{p}\right) \otimes \ell^{p}(X)$ is isomorphic to $\ell^{p}$, thus the claim.

Example 2.4.4. Let $u$ satisfy the condition (1) from Theorem 1.1.1, namely

$$
\int_{c}^{\infty} \frac{d u(t)^{p}}{t^{p}}<\infty
$$

Then $f(t):=u\left(1 / \epsilon_{p}(t)\right)$ satisfies the assumption of Proposition 2.4.2. An example of such $u$ is $u(t)=t \log (t)^{-(1+a) / p}$ for any $a>0$. Indeed,

$$
\begin{aligned}
\int_{c}^{\infty} & \epsilon_{p}(S)^{p} d \frac{1}{\epsilon(S)^{p}\left|\log \left(\epsilon_{p}(t)\right)\right|^{1+a}} \quad\left(t:=\left|\log \left(\epsilon_{p}(S)\right)\right|\right) \\
& =\int_{\left|\log \epsilon_{p}(c)\right|}^{\infty} e^{-p t} d \frac{e^{p t}}{t^{1+a}}=\int_{\left|\log \epsilon_{p}(c)\right|}^{\infty} d t^{-1-a}+p t^{-1-a} d t \\
& =t^{-1-a}-\left.\frac{p}{a} t^{-a}\right|_{\left|\log \epsilon_{p}(c)\right|} ^{\infty}<\infty .
\end{aligned}
$$


2.5. The integrability condition. In this section we will demonstrate the equivalence of the condition (1) from Theorem 1.1.1 and the condition $\left(C_{p}\right)$ introduced by R. Tessera.

For any non-decreasing function $u:[c, \infty) \rightarrow \mathbb{R}_{+}$, let $v(s):=(u(s) / s)^{p}$. Define two functions:

$$
\gamma(s):=\int_{c}^{s} \frac{d\left(u(t)^{p}\right)}{t^{p}}-v(c)
$$

and

$$
\tau(s):=\int_{c}^{s} v(t) d \log (t) .
$$

Observe that both $\gamma$ and $\tau$ are non-decreasing.

Tessera's condition $\left(C_{p}\right)$ says that $\sup \tau<\infty$, while (1) is equivalent to $\sup \gamma<\infty$.

Proposition 2.5.1. Let $u$ be any non-decreasing nonnegative function. Then $\sup \gamma=$ $\sup \tau$.

Proof. Integrating by parts we see that

$$
\gamma(s)=\tau(s)+v(s)
$$

Thus immediately we get that $\sup \gamma \geq \sup \tau$.

To prove the opposite inequality observe that if $\sup \tau=\infty$ then there is nothing left to prove. Assume that $\sup \tau<\infty$. This implies that $\lim \inf v=0$ (moreover, it is an easy exercise, as $u$ is non-decreasing, to replace lim inf by lim, but we shall not use this). Fix $\varepsilon>0$. We can find $s$ such that $v(s) \leq \varepsilon$. Then $\gamma(s) \leq \tau(s)+\varepsilon$. Since $s$ (for a fixed $\varepsilon$ ) can be chosen arbitrarily large we conclude that $\sup \gamma \leq \sup \tau+\varepsilon$. By the arbitrariness of $\varepsilon$ we conclude that $\sup \gamma \leq \sup \tau$.

The Khinchine inequalities provide a bi-Lipschitz (linear) embedding of the Hilbert space into any of the spaces $L^{p}[0,1]$. Therefore, if one is interested in the $L^{p}$-compression, the Hilbert compression provides a lower bound. It is not clear if the same is true for the $\ell^{p}$-compression.

Note that the condition (1) gets slightly weaker as $p$ increases. In particular $u(t)=t / \sqrt[q]{\log (t)}$ satisfies (1) if and only if $p>q$.

2.6. Uniform embeddings of trees. Given any non-decreasing function $u$ with $\int t^{-p} d u(t)^{p}<\infty$, Corollary 2.4.3 and Proposition 2.1.10 provide an embedding of a tree into $\ell^{p}$ space with compression bigger than $u$.

Independently, R. Tessera improved [Tes06, Theorem 7.3] the original construction of E. Guentner and J. Kaminker [GK04, Proposition 4.2] to obtain an embedding of trees with such an asymptotic. 
On the other hand, using the result of J. Bourgain [Bou86, Theorem 1], R. Tessera [Tes06, Corollary 6.3] showed that, for $2 \leq p<\infty$ and any tree $T$ with no vertices of valence 1 or 2 , the compression $\rho$ of any Lipschitz map $T \rightarrow \ell^{p}$ satisfies $\int t^{-p} d \rho(t)^{p}<\infty$.

The difference between our construction and the construction of E. Guentner, J. Kaminker and R. Tessera is the following. The former is a cocycle with respect to the action of the (amenable) stabilizer of the point in the boundary, whereas the latter is a cocycle with respect to the action of the (compact) stabilizer of the vertex in the tree.

By being a cocycle we mean the following property. Let a group $\Gamma$ act on a space $X$. The action induces a representation on the space $W=\ell^{p}(X) \otimes L^{p}([c, \infty))$. The map $\theta: X \rightarrow W$ is called a $\Gamma$-cocycle if $\Gamma \ni \gamma \mapsto \vartheta(\gamma):=\theta(\gamma x)-\gamma \theta(x) \in W$ is independent of $x \in X$. Moreover, then $\vartheta$ satisfies the cocycle relation

$$
\vartheta\left(\gamma \gamma^{\prime}\right)=\gamma \vartheta\left(\gamma^{\prime}\right)+\vartheta(\gamma)
$$

or, in other words, $w \mapsto \gamma w+\vartheta(\gamma)$ is an affine isometric action of $\Gamma$ on $W$.

Proposition 2.6.1. Let a group $\Gamma$ act on a space $X$. If $\xi^{S}: X \rightarrow \mathbb{S}_{1}\left(\ell^{p} X\right)$ are $\Gamma$ equivariant, then the map $\theta$ constructed in Proposition 2.4.2 is a $\Gamma$-cocycle. Moreover, $\vartheta(g)=\theta\left(g x_{0}\right)$ where $x_{0}$ is the chosen reference point.

On the other hand, assume that $\theta$ is a cocycle on the vertex set of a tree with values in a Hilbert space equivariant with respect to some group $\Gamma$, subgroup of the full automorphism group of the tree, with the compression $\rho_{\theta}$ satisfying $\rho_{\theta}(t) / \sqrt{t} \rightarrow \infty$. Then the group $\Gamma$ is necessarily amenable by Theorem 4.1 in [CTV05] or by a small modification of Theorem 5.3 in [GK04].

Note that if $\Gamma$ is compact (e.g. it is the stabilizer of a vertex) then $\vartheta$, being continuous, must be bounded. But this only shows that $\theta$ is bounded on the orbits of $\Gamma$.

\section{Spaces with finite asymptotic dimension}

\subsection{The type function}

Definition 3.1.1. Let $\mathfrak{U}$ be a cover of $X$. Define

(1) the Lebesgue number of $\mathfrak{U}$ at $x \in X$,

$$
L(\mathfrak{U}, x):=\sup _{U \in \mathfrak{U}}\left\{r \mid B_{r}(x) \subset U\right\} ;
$$

(2) the Lebesgue number of $\mathfrak{U}$,

$$
L(\mathfrak{U}):=\inf _{x \in X} L(\mathfrak{U}, x)
$$


(3) the multiplicity of $\mathfrak{U}$ at $x \in X$,

$$
m(\mathfrak{U}, x):=\#\{U \in \mathfrak{U} \mid x \in U\} ;
$$

(4) the multiplicity of $\mathfrak{U}$,

$$
m(\mathfrak{U}):=\max _{x \in X} m(\mathfrak{U}, x)
$$

(5) the mesh of $\mathfrak{U}$,

$$
S(\mathfrak{U}):=\sup \{\operatorname{diam}(U) \mid U \in \mathfrak{U}\} .
$$

Definition 3.1.2 ([DB05]). We say that a metric space $X$ has asymptotic dimension less than $m \in \mathbb{N}$, denoted

$$
\operatorname{asdim} X<m,
$$

if for every $L<\infty$ there exist a number $S<\infty$ and a cover $\mathfrak{U}$ with mesh at most $S$, with multiplicity at most $m$ and with Lebesgue number at least $L$.

Definition 3.1.3. Let $X$ be a metric space of asymptotic dimension at most $m$. Define the type function $D_{m-1}: \mathbb{R}^{+} \rightarrow \mathbb{R}^{+} \cup\{\infty\}$ in the following way: $D_{m-1}(L)$ is the infimum of those $S>0$ for which $X$ can be covered by a family of sets with mesh at most $S$, multiplicity at most $m$ and Lebesgue number at least $L$.

Definition 3.1.4. We also define $\delta_{p}(S)=\sup _{\mathfrak{U}}\left\{\frac{L(\mathfrak{l})}{m(\mathfrak{U})^{2 / p}}\right\}$, where $\mathfrak{U}$ runs over a set of covers of $X$ with mesh at most $S$.

Remark 3.1.5. Of course, $D_{m-1}\left(m^{2 / p} \delta_{p}(S)\right) \geq S$ for any $m$ and $p$.

Remark 3.1.6. The type function was originally ([Gro93, p. 29]) defined in a different way. In the rest of this section we will compare the two definitions.

Proposition 3.1.7. A metric space $X$ has asymptotic dimension at most $k$ iffor every $L$ there exists a cover $\mathfrak{U}=\bigcup_{i=0}^{k} \mathfrak{U}_{k}$ with finite mesh and the property, that, for any $0 \leq i \leq k$, any two different sets from $\mathfrak{U}_{i}$ are $L$-disjoint.

Proof. This is a part of Theorem 1 in [DB05].

The original definition of a type function, which we will call $\widetilde{D}_{k}$, is as follows: $\widetilde{D}_{k}(L)$ is the infimum of the mesh of the covers as in Proposition 3.1.7.

Note that $L / 2$ thickening of the cover as in Proposition 3.1.7 is a cover with mesh at most $\widetilde{D}_{k}(L)+L$, multiplicity at most $k$ and with Lebesgue number at least $L / 2$. Thus

$$
D_{k}(L / 2) \leq \widetilde{D}_{k}(L)+L .
$$


We leave it as an easy exercise to show that if the space is the vertex set of a graph (with unit length edges) with the induced metric, then $\widetilde{D}_{k}(L) \geq L / k-1$. More generally, the inequality $\widetilde{D}_{k}(L) \succeq L$ holds for quasi-geodesic spaces. For such spaces $D_{k} \preceq \widetilde{D}_{k}$ and Theorem 1.1.1 remains true if one replaces $D_{k}$ by $\widetilde{D}_{k}$.

3.2. Asymptotic dimension and property A. In this section we will exhibit a link between the profile $\epsilon_{p}$ and the type function. In particular, we will show that a large class of spaces, namely the spaces with finite asymptotic dimension of linear type, satisfy $\epsilon_{p}(S) \sim S^{-1}$ for all $p \geq 1$.

Theorem 3.2.1. Let $\mathfrak{U}$ be a cover of $X$ with finite multiplicity. Then there exists a map $\xi: X \rightarrow \mathbb{S}_{1}\left(\ell^{p} X\right)$ which is $2 \frac{\sqrt[p]{2 m(\mathfrak{U})^{2}}}{L(\mathfrak{U})}$-Lipschitz and satisfies $S(\xi)=S(\mathfrak{U})$.

Proof. Let $\mathfrak{U}$ be a cover of $X$. For any $U \in \mathfrak{U}$ define

$$
\begin{aligned}
\psi_{U}(x) & :=\operatorname{dist}(x, X-U), \\
\psi(x) & :=\left(\sum_{U \in \mathfrak{U}} \psi_{U}(x)^{p}\right)^{1 / p} .
\end{aligned}
$$

We have $\psi(x) \geq L(\mathfrak{U}, x) \geq L(\mathfrak{U})$, and $\psi_{U}$ is 1-Lipschitz, i.e. $\left|\psi_{U}(x)-\psi_{U}(y)\right| \leq$ $d(x, y)$.

Claim.

$$
\left|\frac{\psi_{U}(x)}{\psi(x)}-\frac{\psi_{U}(y)}{\psi(y)}\right| \leq 2 \frac{(m(\mathfrak{U})+1 / 2)^{1 / p}}{L(\mathfrak{U})} d(x, y)
$$

Indeed,

$$
\left|\frac{\psi_{U}(x)}{\psi(x)}-\frac{\psi_{U}(y)}{\psi(y)}\right|^{p}=\left|\frac{\psi_{U}(x)-\psi_{U}(y)}{\psi(x)}-\frac{\psi_{U}(y)}{\psi(y)} \cdot \frac{\psi(x)-\psi(y)}{\psi(x)}\right|^{p}
$$

(by the inequality between the arithmetic and $p$-mean)

$$
\leq 2^{p-1}\left(\frac{\left|\psi_{U}(x)-\psi_{U}(y)\right|^{p}}{\psi(x)^{p}}+\frac{\psi_{U}(y)^{p}}{\psi(y)^{p}} \cdot \frac{|\psi(x)-\psi(y)|^{p}}{\psi(x)^{p}}\right)
$$

(by the triangle inequality in $\ell^{p}(\mathfrak{U})$ and $\psi_{U}(y) \leq \psi(y)$ )

$$
\begin{aligned}
& \leq \frac{2^{p-1}}{\psi(x)^{p}}\left(\left|\psi_{U}(x)-\psi_{U}(y)\right|^{p}+\sum_{V \in \mathfrak{U}}\left|\psi_{V}(x)-\psi_{V}(y)\right|^{p}\right) \\
& \leq \frac{2^{p-1}}{L(\mathfrak{U})^{p}}(1+2 m(\mathfrak{U})) d(x, y)^{p} .
\end{aligned}
$$


Let $\phi_{U}(x)=\psi_{U}(x) / \psi(x)$ and $\chi_{U}=\psi_{U} /\left\|\psi_{U}\right\|_{p}$. Define

$$
\xi_{x}(z):=\left(\sum_{U \in \mathfrak{U}} \phi_{U}(x)^{p} \chi_{U}(z)^{p}\right)^{1 / p} .
$$

Note that $\chi_{U}$ and $\xi_{x}$ are well defined. The support of $\psi_{U}$ is contained in a ball of radius $S$, thus the norm reduces to the finite sum. Also the sum in the definition of $\xi_{x}(z)$ runs over those $U$ which contain both $x$ and $z$, thus at most $m(\mathfrak{U})$ of them. In particular, $\xi_{x}(z) \neq 0$ if and only if $x$ and $z$ belong simultaneously to at least one $U \in \mathfrak{U}$. Therefore $S(\xi)=S(\mathfrak{U})$.

Moreover,

$$
\begin{aligned}
\left\|\xi_{x}\right\|_{p}^{p} & =\sum_{z \in X} \sum_{U \in \mathfrak{U}} \phi_{U}(x)^{p} \chi_{U}(z)^{p} \\
& =\sum_{U \in \mathfrak{U}} \phi_{U}(x)^{p}\left(\sum_{z \in U} \chi_{U}(z)^{p}\right)=1 .
\end{aligned}
$$

All we have to check is the Lipschitz condition. If $d(x, y) \geq L(\mathfrak{U})$, the condition is trivial as $\left\|\xi_{x}-\xi_{y}\right\| \leq\left\|\xi_{x}\right\|+\left\|\xi_{y}\right\|=2$ for all $x$ and $y$. Therefore assume that $d(x, y)<L(\mathfrak{U})$, and, in particular, there is a set in $\mathcal{U}$ containing both $x$ and $y$. Thus, there are at most $2 m(\mathfrak{U})-1$ sets containing any of $x$ or $z$. As before we check:

$$
\left\|\xi_{x}-\xi_{y}\right\|_{p}^{p}=\sum_{z \in X}\left|\left(\sum_{U \in \mathfrak{U}}\left(\phi_{U}(x) \chi_{U}(z)\right)^{p}\right)^{1 / p}-\left(\sum_{U \in \mathfrak{U}}\left(\phi_{U}(y) \chi_{U}(z)\right)^{p}\right)^{1 / p}\right|^{p}
$$

(by the triangle inequality in $\ell^{p}(\mathfrak{U})$ )

(by the Claim)

$$
\begin{aligned}
& \leq \sum_{z \in X} \sum_{U \in \mathfrak{U}}\left|\phi_{U}(x) \chi_{U}(z)-\phi_{U}(y) \chi_{U}(z)\right|^{p} \\
& =\sum_{U \in \mathfrak{U}}\left|\phi_{U}(x)-\phi_{U}(y)\right|^{p}\left(\sum_{z \in U} \chi_{U}(z)^{p}\right)
\end{aligned}
$$

$$
\begin{aligned}
& \leq \sum_{x \text { or } y \in U \in \mathfrak{U}} 2^{-1}(2 m(\mathfrak{U})+1)\left(\frac{2 d(x, y)}{L(\mathfrak{U})}\right)^{p} \\
& =2^{-1}(2 m(\mathfrak{U})-1)(2 m(\mathfrak{U})+1)\left(\frac{2 d(x, y)}{L(\mathfrak{U})}\right)^{p} \\
& \leq 2 m(\mathfrak{U})^{2}\left(\frac{2 d(x, y)}{L(\mathfrak{U})}\right)^{p}
\end{aligned}
$$

and, in particular, $\xi: X \rightarrow \mathbb{S}_{1}\left(\ell^{p} X\right)$ is $2\left(2 m(\mathfrak{U})^{2}\right)^{1 / p} L(\mathfrak{U})^{-1}$-Lipschitz. 
The proof of Theorem 3.2.1 follows an informal argument of N. Higson and J. Roe [HR00] and more precisely a computation from the proof of Theorem 1 from [DB05] (case $p=1$ of Theorem 3.2.1).

Corollary 3.2.2. Let $X$ be a metric space with $\delta$-function $\delta_{p}$ (see Definition 3.1.4). Then

$$
\epsilon_{p} \leq \frac{2^{1+1 / p}}{\delta_{p}} \leq \frac{4}{\delta_{p}}
$$

for all $1 \leq p<\infty$.

Proof of Theorem 1.1.1. Substitute $f:=u \circ D_{k}^{-1}$ in Corollary 2.4.3. The claim follows from inequalities

$$
4 / \epsilon_{p} \geq \delta_{p} \geq k^{-2 / p} D_{k}^{-1},
$$

due to Remark 3.1.5 and Corollary 3.2.2.

\section{Application to spaces with infinite asymptotic dimension}

4.1. Spaces with polynomial dimension growth. A. Dranishnikov [Dra06] defined groups with a polynomial dimension growth. In terms of the function $\delta_{p}$ this is defined as follows.

Definition 4.1.1. A space has polynomial dimension growth of degree less than $k$ if $\lim _{S \rightarrow \infty} \delta_{2 k}(S)=\infty$.

A straightforward corollary from Corollary 3.2.2 and Proposition 2.1.5 is

Corollary 4.1.2 ([Dra06, Theorem 3.3]). A space with a polynomial dimension growth has property $\mathrm{A}$.

By the very definition, if $p \geq q$, then $\delta_{p} \geq \delta_{q}$. If the space has finite asymptotic dimension then, by Remark 3.1.5, $\lim _{S \rightarrow \infty} \delta_{2 k}(S)=\infty$ for all $p>0$.

Definition 4.1.3. A space is said to have polynomial growth of degree $\alpha$ if there exists a constant $C$ such that every ball of radius $R$ contains at most $C \cdot R^{\alpha}$ elements.

Theorem 4.1.4. A space of polynomial growth of degree $\alpha$ has polynomial dimension growth of degree at most $\alpha+\varepsilon$ for any $\varepsilon>0$ and Hilbert compression rate equal to one. 
Proof. Take a cover by all balls of radius $S / 2$. Its mesh is at most $S$, the Lebesgue number is $S / 2$, and the multiplicity is $C \cdot(S / 2)^{\alpha}$. Thus $\delta_{p}(S) \geq \frac{2^{2 / p-1}}{C^{2 / p}} S^{1-2 \alpha / p}$. In particular, by Corollary 3.2.2, $\epsilon_{p}(S) \leq 4 C^{2} S^{-1+2 \alpha / p}$ and, by Lemma 2.3.2, $\epsilon_{p}(S) \leq\left(4 e^{2 \alpha} C^{2} p^{-1}\right) \log (S) / S$ for $S \geq e^{p}$, and thus the $\ell^{p}$-compression rate for such a space equals one.

A metric space is said to have the doubling property if there exists a constant $K$ such that for all $R>0$ the ball of radius $2 R$ can be covered by $K$ balls of radius $R$. It is an easy observation that such a space has polynomial growth of degree at most $\log _{2} K$.

The compression part of Theorem 4.1.4 for spaces with the doubling property is also a corollary [Ass83, Proposition 2.6] of the beautiful characterization theorem of P. Assouad that $(X, d)$ has the doubling property if and only if $\left(X, d^{\eta}\right)$ admits a bi-Lipschitz embedding into a Euclidean space for all $0<\eta<1$.

4.2. Preliminaries on $\mathbb{Z}^{\boldsymbol{k}}$. Let $\mathbb{A}^{n-1}:=\left\{x=\left(x_{1}, \ldots, x_{n}\right) \in \mathbb{R}^{n} \mid \sum_{i=1}^{n} x_{i}=0\right\}$. Equip $\mathbb{A}^{n-1}$ with a norm $\|x\|:=\sum_{i=1}^{n}\left|x_{i}\right|$. For any $I \subset\{1, \ldots, n\}$ define a functional

$$
\phi_{I}(x):=\frac{\sum_{i \in I} x_{i}}{\# I}-\frac{\sum_{i \notin I} x_{i}}{\# I^{c}}=\frac{n \sum_{i \in I} x_{i}}{\# I \cdot \# I^{c}} .
$$

Here $I^{c}$ denotes the complement of $I$. Note that $\phi_{I}=-\phi_{I^{c}}$.

Remark 4.2.1. For any $I$ we have $\|x\|>\phi_{I}(x)$.

Let

$$
V:=\left\{x \in \mathbb{A}^{n-1} \mid \phi_{I}(x) \leq \frac{1}{2} \text { for all } I\right\}
$$

Let

$$
[i]_{j}:= \begin{cases}i-n & \text { if } j \leq i \\ i & \text { if } j>i .\end{cases}
$$

Define $\Lambda:=\left\{\mathbb{A}^{n-1} \cap \mathbb{Z}^{n}\right\}$. Let $\mathfrak{U}_{i}:=\{[i]+x+V \mid x \in \Lambda\}$.

Lemma 4.2.2. $\mathfrak{U}:=\bigcup_{i=0}^{n-1} \mathfrak{U}_{i}$ is a (closed) cover of $\mathbb{A}^{n-1}$.

Proof. Put for the moment the auxiliary $\ell^{2}$-norm, defined by $\|x\|_{2}=\sqrt{\sum_{i=1}^{n}\left|x_{i}\right|^{2}}$, on $\mathbb{A}^{n-1}$.

Below we will check that $V$ consists of the points that are closer or at the same distance to 0 than to any vector $[i]$ or its image by the action of the permutation group $S_{n}$, which is enough to conclude the claim.

Indeed, the set of points which are closer or at the same distance to 0 than to $[i]$ is defined by the inequality

$$
\langle x \mid[i]\rangle \leq 1 / 2\|[i]\|_{2}^{2},
$$


where $\langle\cdot \mid \cdot\rangle$ is the scalar product associated to $\|\cdot\|_{2}$.

Straightforward computation shows that (2) is equivalent to $\phi_{\{1, \ldots, i\}}(x) \leq 1 / 2$.

One can prove a stronger statement, namely that $V$ is the Voronoi cell of the lattice $\bigcup_{i=0}^{n-1} \Lambda+[i][$ CS99, Ch. 6.6 and 21.3.B].

Lemma 4.2.3. Each $\mathfrak{U}_{i}$ consists of $1 /(n-1)$-disjoint sets.

Proof. Let $x$ and $x^{\prime}$ be two different points in $[i]+\Lambda$. Choose $i_{0}$ such that $x_{i_{0}}>x_{i_{0}}^{\prime}$. Let $I=\left\{i_{0}\right\}$. Then

$$
\phi_{I}\left(x-x^{\prime}\right) \geq \frac{n\left(x_{i_{0}}-x_{i_{0}}^{\prime}\right)}{1 \cdot(n-1)} \geq \frac{n}{n-1} .
$$

Let $z \in x+V$ and $z^{\prime} \in x^{\prime}+V$. Then

$\left\|z-z^{\prime}\right\| \geq \phi_{I}\left(z-z^{\prime}\right) \geq \phi_{I}\left(x-x^{\prime}\right)-\phi_{I}(z-x)+\phi_{I}\left(z^{\prime}-x^{\prime}\right) \geq \frac{n}{n-1}-\frac{1}{2}-\frac{1}{2}=\frac{1}{n-1}$,

by Remark 4.2.1.

Question 4.2.4. What is the best estimate for the disjointness?

The set of extremal points (the vertices) of $V$ is the orbit of $\sigma=(2 n)^{-1}(1-n$, $3-n, \ldots, n-1)$ under the permutations of coordinates [CS99, Chapter 6.6 and 21.3.B]. From now on assume that $n=2 k$ is even. Then $\|\sigma\|=(4 k)^{-1} \cdot 2 \sum_{i=1}^{k}(2 i-1)=$ $k / 2$.

Thus we have proved

Lemma 4.2.5. The $\frac{1}{2(2 k-1)}$ open thickening $\mathfrak{U}^{\prime}$ of $\mathfrak{U}$ satisfies $L\left(\mathfrak{U}^{\prime}\right) \geq \frac{1}{2(2 k-1)}$, $m\left(\mathfrak{U}_{k}^{\prime}\right)=2 k$ and $S\left(\mathfrak{U}^{\prime}\right)=k+1 /(2 k-1)$.

Corollary 4.2.6. $D_{2 k ; \mathbb{Z}^{k}}(L) \leq\left(2 k^{2}-2 k+1\right) L$.

Proof. Observe that the map $\iota: \mathbb{Z}^{k} \ni\left(\ldots, z_{m}, \ldots\right) \mapsto(1 / 2)\left(\ldots, z_{m},-z_{m}, \ldots\right) \in$ $\mathbb{A}^{2 k-1}$ is an isometry. Thus the induced cover $\iota^{*} \mathfrak{U}^{\prime}$ satisfies the inequality of the claim for $L=\frac{1}{2(2 k-1)}$.

Since we can precompose $\iota$ with a homotety of $\mathbb{A}^{n-1}$ the claim is true for arbitrary $L$.

Question 4.2.7. What is the rate of $D_{k ; \mathbb{Z}^{k}}$ ? Can one replace a square in $D_{2 k ; \mathbb{Z}^{k}}(L) \leq$ $2 k^{2} L$ by some lower power? What about the estimates for $D_{k^{\alpha} ; \mathbb{Z}^{k}}$ ? 
One can adjust the results of the next section to prove that if $D_{c k^{\alpha} ; \mathbb{Z}^{k}}(L) \leq C k^{\beta} L$ then $\epsilon_{p ; \mathbb{Z} \imath \mathbb{Z}} \preceq \log (S) / \sqrt[1+\beta]{S}$ and, what follows, the compression rate of $\mathbb{Z} \imath \mathbb{Z}$ is at least $\frac{1}{1+\beta}$. On the other hand G. Arzhantseva, V. Guba and M. Sapir [AGS04, Theorem 1.8] showed that the Hilbert compression rate of $\mathbb{Z} \geq \mathbb{Z}$ is at most $3 / 4$. It follows that $D_{c k^{\alpha}}(L) \leq k^{\beta} \cdot L$ is impossible with $\beta<1 / 3$. Thus the answer to Question 4.2.7 is nontrivial.

4.3. The lamplighter group $\mathbb{Z} \geq \mathbb{Z}$. The lamplighter group is the (restricted) wreath product $\mathbb{Z} \imath \mathbb{Z}$, where $H \imath G$ is defined as the semidirect product $\bigoplus_{G} H \rtimes G$, where $G$ acts on $\bigoplus_{G} H$ by permutation of the factors. In other words

$$
H \prec G=\langle H, G \mid[a,[b, g]]=[a, b], a, b \in H, g \in G-\{e\}\rangle .
$$

Proposition 4.3.1 ([Dra06, Prop 4.2]). Let $K$ be a normal subgroup of $G$. Let $G$ be equipped with a (left invariant) metric. Put on $K$ the restricted metric, and induce the metric on $H=G / K$. Let $k$ and $h$ be two integers. Then

$$
D_{k h-1 ; G}(L) \leq \frac{4}{3} D_{k-1 ; K}\left(6 D_{h-1 ; H}(L)\right) .
$$

In our case $G=\mathbb{Z} \imath \mathbb{Z}$ and $H=\mathbb{Z}$. Thus $D_{2 k-1 ; G}(L) \leq 4 / 3 D_{k-1 ; K}(24 L)$, where $K=\bigoplus_{\mathbb{Z}} \mathbb{Z}$ with the restricted metric $d_{K}$. This translates to the following statement. Given a cover $\mathfrak{V}$ of $K$ one constructs a cover $\mathfrak{W}$ of $\mathbb{Z} \imath \mathbb{Z}$ such that $L(\mathfrak{W}) \geq L(\mathfrak{V}) / 24, S(\mathfrak{W}) \leq 4 / 3 S(\mathfrak{V})$ and $m(\mathfrak{W}) \leq 2 m(\mathfrak{U})$.

Following A. Dranishnikov [Dra06] we decompose $K:=\bigoplus_{\mathbb{Z}} \mathbb{Z}=K_{m} \oplus K^{m}$, where

$$
K_{m}=\bigoplus_{\{-m+1,-m+2, \ldots, m-1\}} \mathbb{Z} \text { and } K^{m}=\bigoplus_{\{\ldots,-m-1,-m, m, m+1, \ldots\}} \mathbb{Z} .
$$

Lemma 4.3.2. Let $K / K_{m} \ni \alpha \rightarrow g_{\alpha} \in K$ be a section and let $\mathfrak{Y}$ be a cover of $K_{m}$. Then $\widetilde{\mathfrak{V}}:=\bigcup_{\alpha} g_{\alpha} \mathfrak{V}$ is a cover of $K$ with the same mesh and multiplicity. Moreover $L(\widetilde{\mathfrak{V}})=\min \{L(\mathfrak{V}), 2 m+1\}$.

Proof. Observe that any two cosets of $K_{m}$ are at least $2 m+1$ apart.

Proposition 4.3.3. Let $f:(G, d) \rightarrow\left(G^{\prime}, d^{\prime}\right)$ be a map, and $\mathcal{U}$ be a covering of $G^{\prime}$ then

$$
\rho_{+}\left(L\left(f^{*} \mathfrak{U}\right)\right) \geq L(\mathfrak{U}), \quad \rho_{-}\left(S\left(f^{*} \mathfrak{U}\right)\right) \leq S(\mathfrak{U}),
$$

where $f^{*} \mathfrak{U}=\left\{f^{-1} U \mid U \in \mathfrak{U}\right\}$ is the induced cover of $G$, and $\rho_{ \pm}$are the compression and dilation of $f$. 
Lemma 4.3.4. The natural homomorphism $j: K_{m} \rightarrow \mathbb{Z}^{2 m-1}$ satisfies

$$
d_{K}(x, y)-4(m-1) \leq d_{1}(f(x), f(y)) \leq d_{K}(x, y) .
$$

Proof. Recall ([CT05]) that if $f: \mathbb{Z} \rightarrow \mathbb{Z}$ is an element of $K$ (i.e. if $f$ has finite support), then the length of $f$ (with respect to the metric restricted from $\mathbb{Z} \backslash \mathbb{Z}$ ) is equal to

$$
2 \max (\{0\} \cup\{k \mid f(k) \neq 0\})+2 \max (\{0\} \cup\{-k \mid f(k) \neq 0\})+\sum_{k \in \mathbb{Z}}|f(k)| .
$$

Corollary 4.3.5. There exists a cover $\mathfrak{Y}$ of $K_{m}$ satisfying $L(\mathfrak{V}) \geq 2 m$, $S(\mathfrak{V}) \leq 16 m^{3}$ and $m(\mathfrak{V}) \leq 4 m$.

Proof. By Corollary 4.2.6 and the previous lemma we may construct a cover of $K_{m}$ satisfying the bounds. The bound on mesh follows from

$$
\begin{aligned}
S(\mathfrak{V}) & \leq D_{4 m ; \mathbb{Z}^{2 m}}(2 m)+4(m-1) \\
& \leq\left(2(2 m)^{2}-2(2 m)+1\right) \cdot(2 m)+4(m-1) \leq 16 m^{3} .
\end{aligned}
$$

Let $m=12 L$. By the result of Dranishnikov, we construct a cover $\mathfrak{W}$ of $\mathbb{Z} 々 \mathbb{Z}$ with the properties $L(\mathfrak{W}) \geq \frac{1}{24} \cdot 2 \cdot 12 L=L, S(\mathfrak{W}) \leq 4 / 3 \cdot 16(12 L)^{3}=36864 L^{3}$ and $m(\mathfrak{W}) \leq 2 \cdot 4 \cdot 12 L=96 L$.

Corollary 4.3.6. $\delta_{p ;} \mathbb{Z} \geq \mathbb{Z}(S) \geq(96)^{-2 / p}\lfloor\sqrt[3]{S / 36864}\rfloor^{1-2 / p} \geq(1 / C) S^{1 / 3-2 /(3 p)}$. In particular, the group $\mathbb{Z} \imath \mathbb{Z}$ has asymptotic dimension growth of order $1+\varepsilon$ for any $\varepsilon>0$.

Corollary 4.3.7. $\epsilon_{p ; \mathbb{Z} \geq \mathbb{Z}}(S) \preceq \log (S) / \sqrt[3]{S}$ for any $1 \leq p<\infty$.

Proof. This follows from Corollary 3.2.2 and Lemma 2.3.2

Corollary 4.3.8. The $\ell^{p}$-compression rate of E. Guentner and J. Kaminker of $\mathbb{Z} \imath \mathbb{Z}$ is at least $1 / 3$ for all $1 \leq p<\infty$.

Remark 4.3.9. This result is not sharp. By other techniques G. Arzhantseva, V. Guba and M. Sapir [AGS04, Theorem 1.8] and independently Y. Stalder and A. Valette [SV06, Corollary 4.5a] showed that the Hilbert compression rate is at least 1/2. Recently R. Tessera showed that the Hilbert compression rate is at least 2/3 [Tes06, Corollary 15].

On the other hand P. Nowak showed [Now07, Corollary 4.4] that $\epsilon_{1 ;} \backslash \mathbb{Z} \sim S^{-1}$, thus the $\ell^{1}$-compression rate of $\mathbb{Z} \geq \mathbb{Z}$ is one. By the Hölder property of the Mazur map the $\ell^{p}$-compression rate is at least $1 / p$, which gives a better estimate than Corollary 4.3 .8 for $1 \leq p<3$. 


\section{References}

[ADS06] Goulnara Arzhantseva, Cornelia Druţu, and Mark Sapir, Compression functions of uniform embeddings of groups into Hilbert and Banach spaces. Preprint 2006, arXiv:math.GR/0612378.

[AGS04] Goulnara Arzhantseva, Victor Guba, and Mark Sapir, Metrics on diagram groups and uniform embeddings in a Hilbert space. Comment. Math. Helv. 81 (2006), 911-929. Zbl 05071527 MR 2271228

[Ass82] Patrice Assouad, Sur la distance de Nagata, C. R. Acad. Sci. Paris Sér. I Math. 294 (1982), 31-34. Zbl 0481.54015 MR 0651069

[Ass83] Patrice Assouad, Plongements lipschitziens dans $\mathbf{R}^{n}$. Bull. Soc. Math. France 111 (1983), 429-448. Zbl 0597.54015 MR 0763553

[DB05] George Bell and Alexander Dranishnikov, Asymptotic groups in Bȩdlewo. Preprint 2005. arXiv:math.GR/0507570.

[BL00] Yoav Benyamini and Joram Lindenstrauss, Geometric nonlinear functional analysis. Amer. Math. Soc. Colloq. Publ. 48, Amer. Math. Soc., Providence, RI, 2000. Zbl 0946.46002 MR 1727673

[BS00] M. Bonk and O. Schramm, Embeddings of Gromov hyperbolic spaces. Geom. Funct. Anal. 10 (2000), 266-306. Zbl 0972.53021 MR 1771428

[Bou86] J. Bourgain, The metrical interpretation of superreflexivity in Banach spaces. Israel J. Math. 56 (1986), 222-230. Zbl 0643.46013 MR 0880292

[BS05] Sergei Buyalo and Viktor Schroeder, A product of trees as universal space for hyperbolic groups. Preprint 2005. arXiv:math.GR/0509355.

[CS99] J. H. Conway and N. J. A. Sloane, Sphere packings, lattices and groups. Third edition, Grundlehren Math. Wiss. 290, Springer-Verlag, New York 1999. Zbl 0915.52003 MR 1662447

[CT05] Sean Cleary and Jennifer Taback, Dead end words in lamplighter groups and other wreath products. Quart. J. Math. Oxford Ser. (2) 56 (2005), 165-178. Zbl 02230593 MR 2143495

[CTV05] Yves de Cornulier, Romain Tessera, and Alain Valette, Isometric group actions on Hilbert spaces: growth of cocycles. Geom. Funct. Anal. 17 (2007), 770-792. Zbl 05207474

[DJ99] A. Dranishnikov and T. Januszkiewicz, Every Coxeter group acts amenably on a compact space. Topology Proc. 24 (1999), 135-141. Zbl 0973.20029 MR 1802681

[Dra06] Alexander Dranishnikov, Groups with a polynomial dimension growth. Geom. Dedicata 119 (2006), 1-15. Zbl 05046849 MR 2247644

[FM98] Benson Farb and Lee Mosher, A rigidity theorem for the solvable Baumslag-Solitar groups. With an appendix by Daryl Cooper, Invent. Math. 131 (1998), 419-451. Zbl 0937.22003 MR 1608595

[GK04] Erik Guentner and Jerome Kaminker, Exactness and uniform embeddability of discrete groups. J. London Math. Soc. (2) 70 (2004), 703-718. Zbl 1082.46049 MR 2160829 
[Gro93] M. Gromov, Asymptotic invariants of infinite groups. In Geometric group theory (Sussex, 1991), London Math. Soc. Lecture Note Ser. 182, Vol. 2, Cambridge University Press, Cambridge 1993, 1-295. Zbl 0841.20039 MR 1253544

[HR00] Nigel Higson and John Roe, Amenable group actions and the Novikov conjecture. J. Reine Angew. Math. 519 (2000), 143-153. Zbl 0964.55015 MR 1739727

[Jan02] Tadeusz Januszkiewicz, For Coxeter groups $z^{|g|}$ is a coefficient of a uniformly bounded representation. Fund. Math. 174 (2002), 79-86. Zbl 1038.20025 MR 1925487

[LS05] Urs Lang and Thilo Schlichenmaier, Nagata dimension, quasisymmetric embeddings, and Lipschitz extensions. Internat. Math. Res. Notices 2005 (58) (2005), 3625-3655. Zbl 1095.53033 MR 2200122

[Maz29] Stanisław Mazur, Une remarque sur l'homéomorphisme des champs fonctionnels. Studia Math. 1 (1929), 83-85, 1930. JFM 55.0242.01

[Now07] Piotr Nowak, On exactness and isoperimetric profiles of discrete groups. J. Funct. Anal. 243 (2007), 323-344. Zbl 05129777 MR 2291440

[SV06] Yves Stalder and Alain Valette, Wreath products with the integers, proper actions and Hilbert space compression. Geom. Dedicata 124 (2007), 199-211. Zbl 05180056 MR 2318545

[Tes06] Romain Tessera, Asymptotic isoperimetry on groups and uniform embeddings into Banach spaces. Preprint 2006. arXiv:math.GR/0603138.

[Tes07] Romain Tessera, Quantitative property A, Poincaré inequalities, $L^{p}$-compression and $L^{p}$-distortion for metric measure spaces. Preprint 2007. arXiv:math.MG/0702384.

[Yu98] Guoliang Yu, The Novikov conjecture for groups with finite asymptotic dimension. Ann. of Math. (2) 147 (2) (1998), 325-355. Zbl 0911.19001 MR 1626745

[Yu00] Guoliang Yu, The coarse Baum-Connes conjecture for spaces which admit a uniform embedding into Hilbert space. Invent. Math. 139 (2000), 201-240. Zbl 0956.19004 MR 1728880

Received July 18, 2006; revised February 22, 2007

Ś. R. Gal, Mathematical Institute, University of Wrocław, pl. Grunwaldzki 2/4, 50-384 Wrocław, Poland

E-mail: sgal@math.uni.wroc.pl 